\title{
QUANTITATIVE TRAIT LOCI (QTLS) MAPPING FOR SALT STRESS TOLERANCE IN WHEAT AT GERMINATION STAGE
}

\author{
Nazima Batool ${ }^{1}$, Noshin Ilyas ${ }^{1, *}$, Armghan Shahzad ${ }^{2, *}$, Bernard A. Hauser $^{3}$ and Muhammad \\ Arshad ${ }^{1}$
${ }^{1}$ Department of Botany, Pir Mehr Ali Shah Arid Agriculture University Rawalpindi, Pakistan; ${ }^{2}$ National Institute for Genomics and Advanced Biotechnology, NARC, Islamabad, Pakistan; ${ }^{3}$ Department of Biology, College of Arts and Science, University of Florida, USA.
*Corresponding author's e-mail: noshinilyas@yahoo.com

\begin{abstract}
Identification of genetic factors responsible for controlling germination traits under salinity will allow developing tolerant cultivars. Here we have studied quantitative trait loci (QTL) linked to seed germination and early seedling growth traits using recombinant inbred lines generated from crossing of wheat cultivars, Pasban 90 (salt tolerant) and Frontana (salt susceptible). QTL analysis were done using composite interval mapping (CIM) for germination percentage (G\%), germination index (GI), seedling vigor index (SVI), shoot length (SL), root length (RL), fresh wight (FW) \& dry weight (DW). In this QTLs analysis total 44 QTLs were identified, 26 QTLs out of 44 were identified in $150 \mathrm{mM}$ salt stressed and eighteen QTLs under control environment. Eleven main quantitative trait loci (QTLs) were localized on 1B, 2B, 3B, 5B, 6B and 7B chromosome of mapping population in stressed environment. QTLs mapped on 6A, 3B, 6B and 6D chromosome for root and shoot length, fresh and dry weight were recognized as a major QTLs under control treatment. QTLs for root and shoot length were mapped on 2D same position in xgdm36-xwmc111. Root length and dry weight QTLs was flanked in interval of xgwm70-xgwm325 markers on 5B chromosomes. The result of this study revealed that QTLs play main role in salt tolerance at seed germination and early seedling growth stage.
\end{abstract}

Keywords: Salinity, germination attributes, QTLs, shoot length, 5B chromosomes.

\section{INTRODUCTION}

Various abiotic stress factors impose negative effect on plant growth and productivity (Shahzad et al., 2012), among them salt stress is one of major factor that drastically reduce crop plant growth and development (Azadi et al., 2015). Salinity affects $800 \mathrm{mh}$ land area in arid and semi-arid environment (Ahmad et al., 2013). Wheat (Triticum aestivum) is staple food and primary crop plant in Pakistan (Batool et al., 2016). Farmers in Pakistan grow wheat in an area nine thousand hectares and 3.1\% contribution in an annual GDP. Although enhancement of wheat tolerance for salt stress is main concern but, salt tolerance through physiological, biochemical and genetic mechanism is complex trait (Genc et al., 2010). Wheat plant exhibits many QTLs that enhance salt tolerance in this species (Ma et al., 2007; Munns and Tester, 2008). Genetic markers development revealed genetic architecture of salt tolerance trait. This help to analyzed individual QTLs playing role in salt tolerance (Ghaedrahmati et al., 2014). Number of scientist mapped QTLs related to salt tolerance mainly linked with morphological and yield trait (ElHendawy et al., 2009; Genc et al., 2010; Amin and Ayman, 2013; Mahdinejad et al., 2014; Azadi et al., 2015). Present study differs from above mention studies because it identifies QTLs in wheat mapping population connected to salt tolerance at germination and early seedling growth stage. Shahzad et al. (2012) describes that seed germination and seedling growth is utmost vulnerable phase to salinity and germination ability of wheat plant $\&$ is controlled by many genes (Song et al., 2005). In order to develop salt tolerant line it is very important to identify salt tolerance mechanism at seedling stage. Simple sequence repeats (SSR) are being used in present study to categorize QTLs linked to salinity tolerance at seed germination and early seedling growth stage.

\section{MATERIALS AND METHODS}

The study was conducted at National Agriculture Research Center (NARC), Islamabad Pakistan and Department of Biology, University of Florida, USA. Pasban90 and Frontana were used to generate a mapping population mixture of salt tolerant and salt susceptible recombinant inbred lines (RILs). Mapping population comprised of eighty seven (F7) RILs and evaluated at germination and seedling stge. Pasban-90 shows tolerance toward salt stress but Frontana is salt susceptible (Ahmad et al., 2013). Complete randomized design with three replications was used for this trial. Surface sterilization of RILs and parental seeds were done using 1\% sodium hypochlorite solution for 10 minute and distilled water used for washing. Ten seeds were germinated in petri plates using 
method describe by Munns and James (2003). During seed germination and propagation, photoperiod 18/10 day/night, temperature $26 / 19^{\circ} \mathrm{C}$ day/night \& relative humidity 65 percent was maintained. All seeds of RILs and parentes were evaluated against control growth and salt stress (supplemented with $150 \mathrm{mM} \mathrm{NaCl}$ ). For salt stress induction, apply $3 \mathrm{ml}$ of sodium chloride was applied to all petri-plates on second day for 10 days. RILs and parental seeds were considered germinated when a seedling length was $5 \mathrm{~mm}$ (Nichols et al., 2009).

Data for following germination attributes such as germination percentage, germination index and seedling vigor index were measured. Mirza et al. (2015) method was used to calculate germination percentage and germination index was calculated by using ISTA (2005). Seedling vigor index was recorded by Abdulbaki and Anderson (1973) method. For early seedling growth following parameter was recorded such as shoot and root length, fresh and dry weight (Mirza et al., 2015). Sharp et al. (1988) describe a procedure to extract DNA from young leaves of RILs and Parents was used. Two hundred and eighty SSR markers were used to check polymorphism among parents, 202 markers out of 280 show polymorphism with parents were applied on mapping population. SSR markers sequence record was used from GrainGene database (http://wheat.pw.usda.gov) WMC (wheat microsatellite consortium) (Guyomarc'h et al., 2002 (CFA \& CFD); Somers et al., 2004), BARC (Beltsville Agricultural Research Center) (Song et al., 2005) and GWM (Gatersleben wheat microsatellites) (Roder et al., 1998). Polymerase chain reaction (PCR) used for DNA amplification reaction using SSR markers, initial denaturation at $94^{\circ} \mathrm{C}$ for $4 \mathrm{~min}, 35-40$ cycles was carried out at $94^{\circ} \mathrm{C}$ for $45 \mathrm{sec}$, for primer annealing $45 \mathrm{sec}$ at 55 to $60^{\circ} \mathrm{C}$ and $45 \mathrm{sec}$ for DNA amplification at $72^{\circ} \mathrm{C}, 10 \mathrm{~min}$ for DNA final extension at $72^{\circ} \mathrm{C}$ and $2 \%$ agarose gel was used to separate PCR and ethidium bromide was used for visualization.

After gel band scoring, word data files were generated in which locus A, B and - were scored as a genotype. Linkage map was constructed using Kosambi mapping function by map maker ver. 3.0. Twenty one linkage groups were identified by comparing earlier maps in literature (Somar et $a l ., 2004)$. After linkage map construction, QDF data file generated by using excel sheets in which phenotypic and genotypic data was combined. QDF data files were subjected to QGene ver. 4.0 to identify QTLs using composite interval mapping and logarithm of odds (LOD) 2.5 thresholds. QTLs clustering were analyzed on chromosomes by using map chart ver. 2.0 and statistical evaluation was done by using STATISTIX.

\section{RESULTS}

Phenotypic variation: Seed germination and seedling growth phase is most important stage in wheat plant growth and development. Salt stress imposed negative impact on wheat seed germination and seedling growth. Recombinant inbred lines studied traits show normal distribution under salt stress condition. RILs were significantly $(\mathrm{p} \leq 0.05)$ different for each trait under $150 \mathrm{mM}$ salt stress. RILs distribution show high and low transgressive segregation for all recorded data. There was a closer distribution of mapping population for germination percentage and germination index under salt stress (Fig. 1-2).

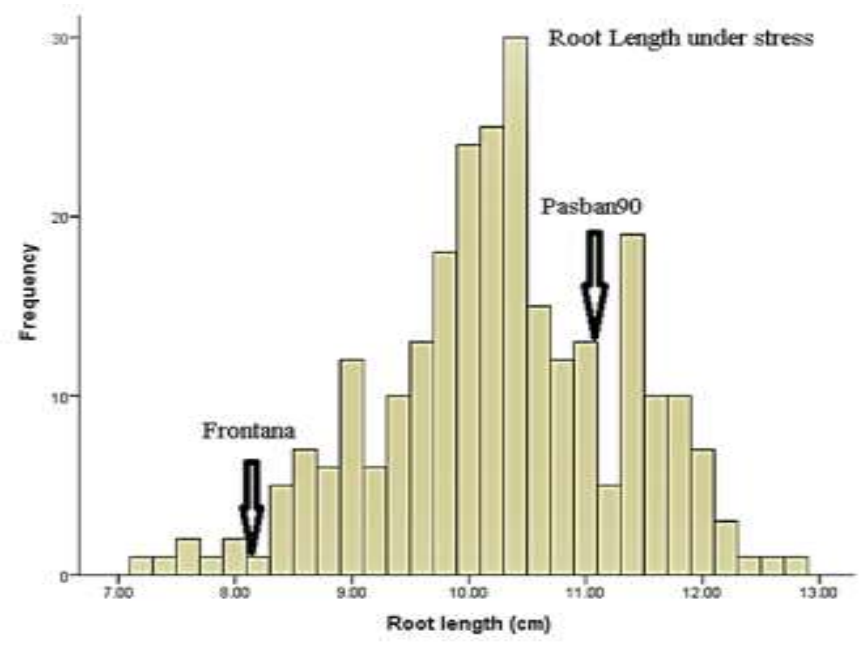

Figure 1. Frequency distribution of traits and parent position among RILs under salt stress.

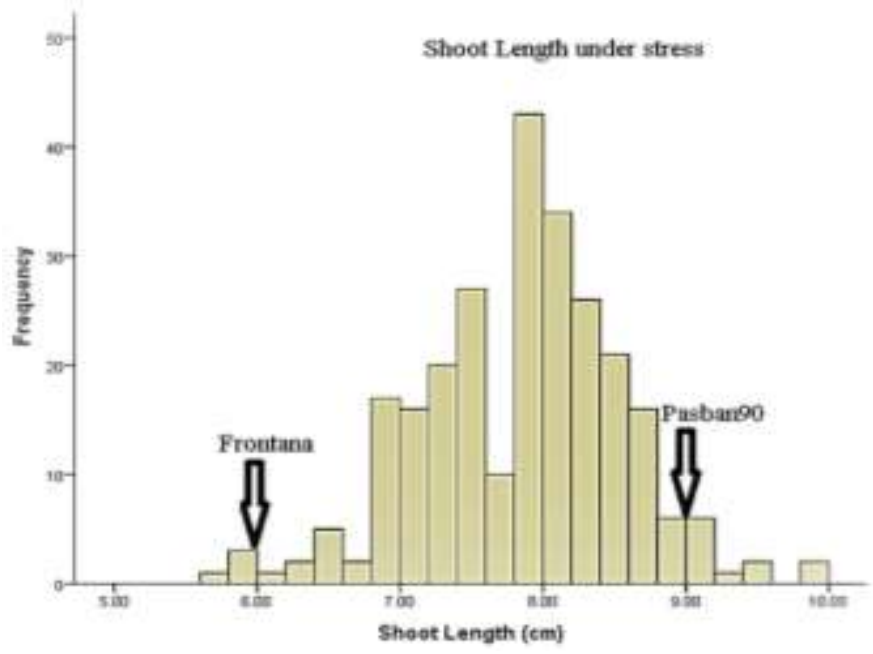

Figure 2. Frequency distribution of traits and parent position among RILs under salt stress.

Parents (Pasban 90 and Frontana) were lies far from each other in stress condition. The phenotypic variation in seedling vigor index (SVI), root length (RL), shoot length (SL), fresh weight (FW) and dry weight (DW) show wide distribution among mapping population under salt stress was observed 
(Fig 3-7). Pasban 90 have shown higher value for all traits as compared to Frontana under salt stress environment. Correlation co-efficient analysis was recorded for all studied traits from values of non-stressed and salt stress conditions (Table 1). A significant correlation was measured between root length and shoot length under control and salt stress condition. Positive correlation was recorded under salt stress environment but most significant correlation among root length, fresh weight and dry weight $(\mathrm{p} \leq 0.05)$.

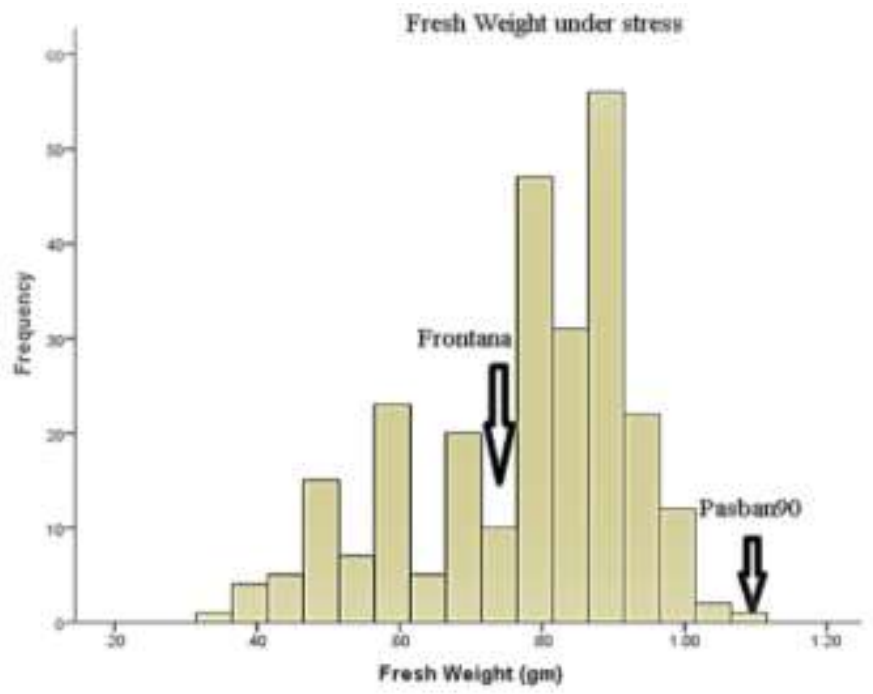

Figure 3. Frequency distribution of traits and parent position among RILs under salt stress.

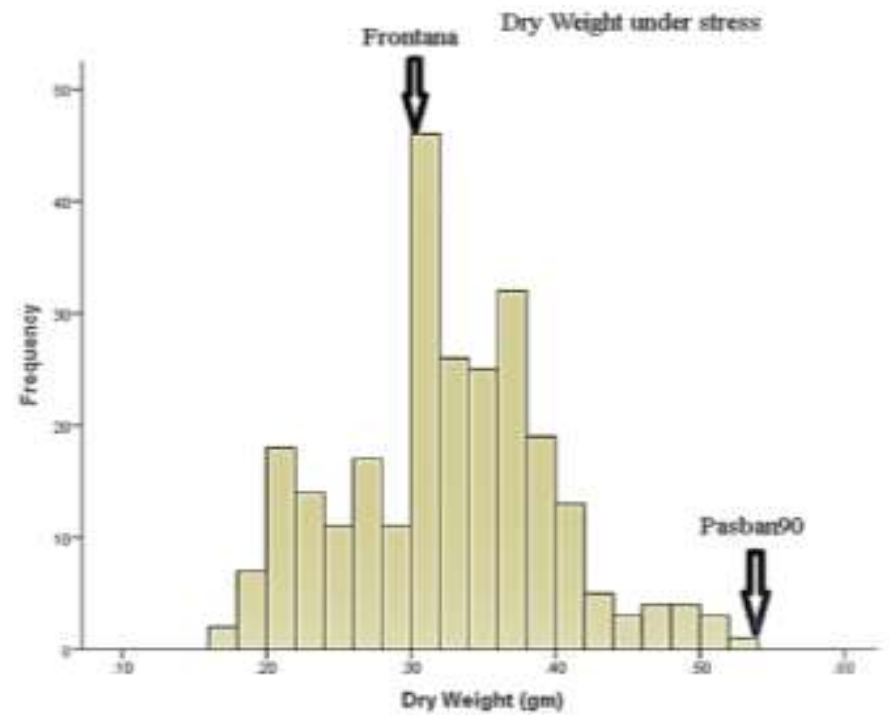

Figure 4. Frequency distribution of traits and parent position among RILs under salt stress.

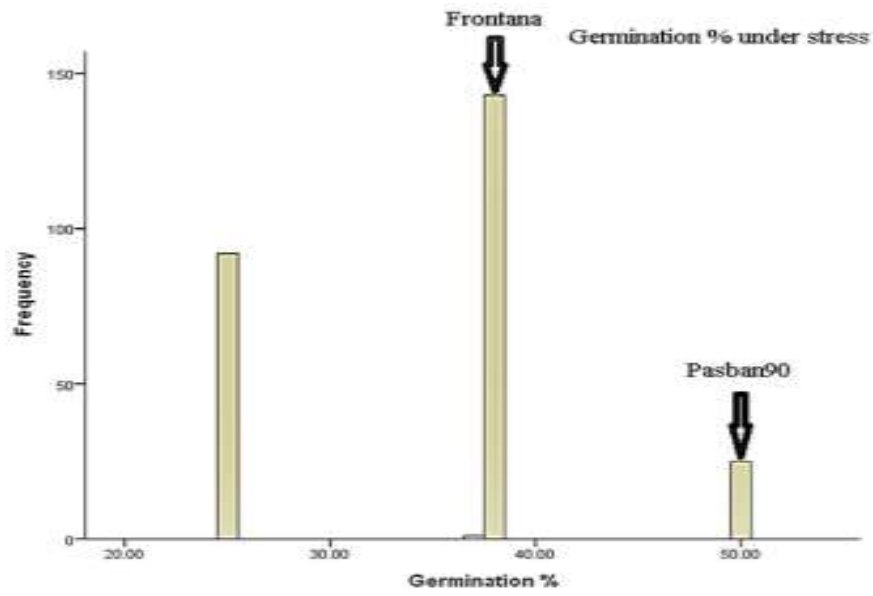

Figure 5. Frequency distribution of traits and parent position among RILs under salt stress.

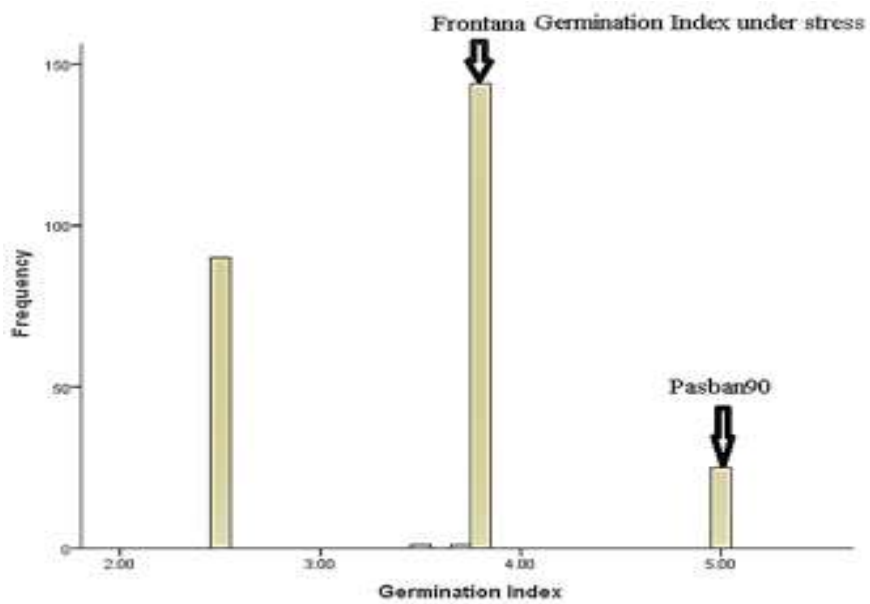

Figure 6. Frequency distribution of traits and parent position among RILs under salt stress.

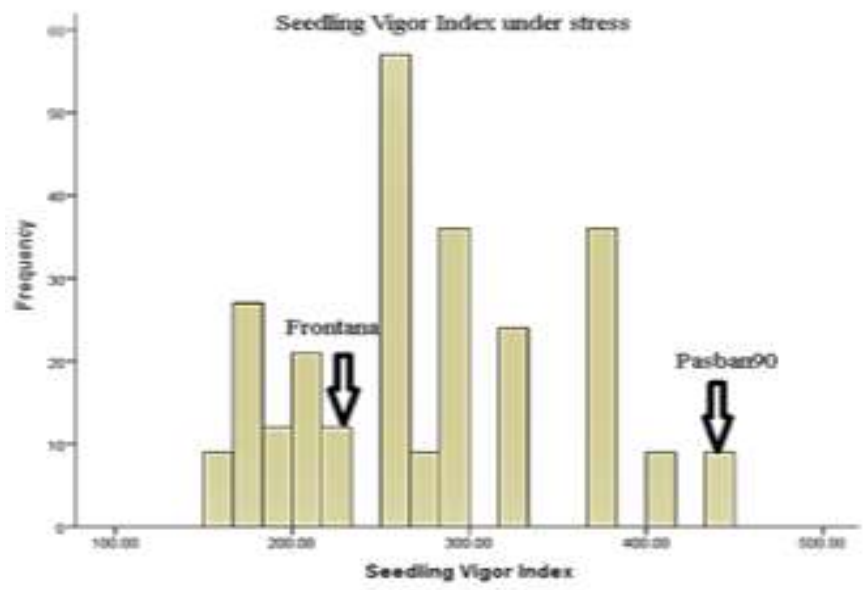

Figure 7.Frequency distribution of traits and parent position among RILs under salt stress. 
Table 1. Correlation coefficients between traits measured on the Parents and RILs under control and salt stress.

\begin{tabular}{|c|c|c|c|c|c|c|c|c|}
\hline & & RL & SL & FW & DW & G\% & GI & SVI \\
\hline Root & Con & 1.00 & & & & & & \\
\hline Length & Str & 1.00 & & & & & & \\
\hline Shoot & Con & $0.61 * *$ & 1.000 & & & & & \\
\hline Length & Str & $0.57 *$ & 1.000 & & & & & \\
\hline Fresh & Con & $0.35^{*}$ & $0.340^{*}$ & 1.000 & & & & \\
\hline Weight & Str & $0.33 * *$ & $0.510 *$ & 1.000 & & & & \\
\hline Dry & Con & $0.35 *$ & 0.390 & $0.510 * *$ & 1.000 & & & \\
\hline Weight & Str & $0.46^{*}$ & $0.290 *$ & $0.280 *$ & 1.000 & & & \\
\hline Germination & Con & $0.39 *$ & $0.029^{\mathrm{ns}}$ & $0.010^{\mathrm{ns}}$ & $0.029 *$ & 1.00 & & \\
\hline Percentage & Str & $0.41 *$ & $-0.014^{\mathrm{ns}}$ & $0.029^{\text {ns }}$ & $0.390 *$ & 1.00 & & \\
\hline Germination & Con & $0.55^{*}$ & $0.041^{\mathrm{ns}}$ & $-0.010^{\text {ns }}$ & $0.030^{\mathrm{ns}}$ & $0.91 *$ & 1.00 & \\
\hline Index & Str & $0.29 *$ & $-0.019^{\text {ns }}$ & $0.023^{\text {ns }}$ & $0.340 *$ & $0.92 *$ & 1.00 & \\
\hline Seedling & Con & $0.54 *$ & $0.089^{\text {ns }}$ & $-0.010^{\mathrm{ns}}$ & 0.031 & $0.99 *$ & $1.00 * *$ & 1.00 \\
\hline Vigor Index & Str & $0.32 *$ & $-0.031^{\mathrm{ns}}$ & $0.012^{\mathrm{ns}}$ & $0.330 *$ & $0.90 * *$ & $0.95^{* *}$ & 1.00 \\
\hline
\end{tabular}

Significance levels: ${ }^{\mathrm{ns}}$ non-significant, $* \mathrm{P}=0.05, * * \mathrm{P}=0.01$.

Composite interval mapping: In total 280 SSR markers were applied to mapping population and out of 280 SSR markers 202 were polymorphic. Chi- square test was used to confirm 1:1 ratio and twenty one linkage groups were identified. Total forty four QTLs were mapped on 16 chromosomes under control and salt stress condition. QTLs were recorded using composite interval mapping (CIM) on sixteen out of twenty one linkage groups for all traits under control and $150 \mathrm{mM}$ stressed environment with logarithm of odds (LOD) value ranging from 2.5 to 4.1 (Fig. 8-15).
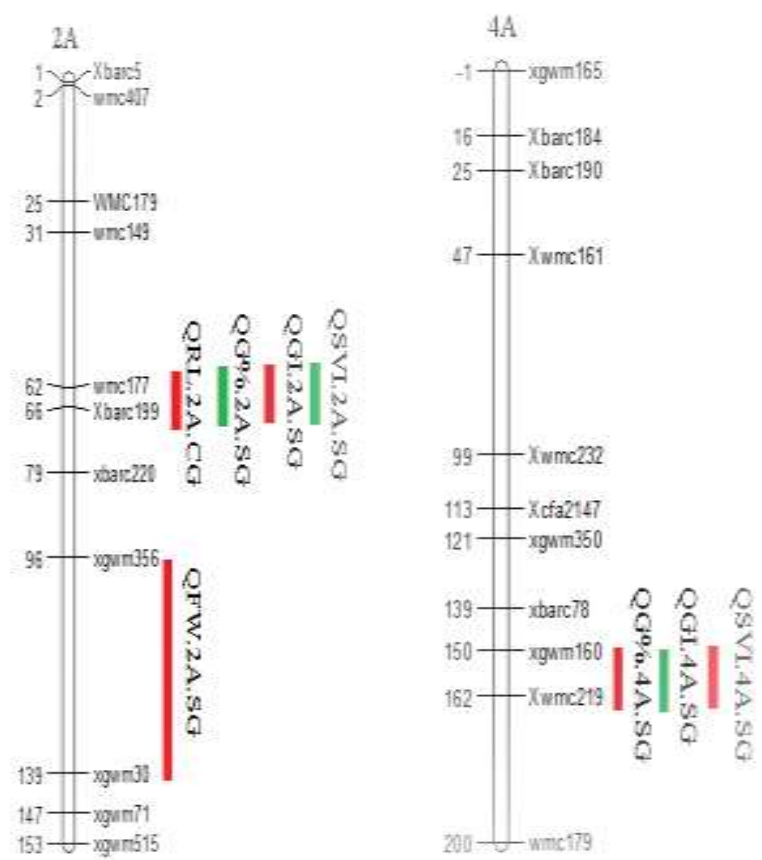

Figure 8. QTL identified on $2 \mathrm{~A}$ and $4 \mathrm{~A}$ chromosome of recombinant inbred lines in non-stressed and stressed conditions.
QTLs were mapped in control and stressed environment which might be permitting the difference between constitutive and adaptive reactions. Mostly QTLs were recorded in stressed environment and mainly contributes for seedling growth trait.

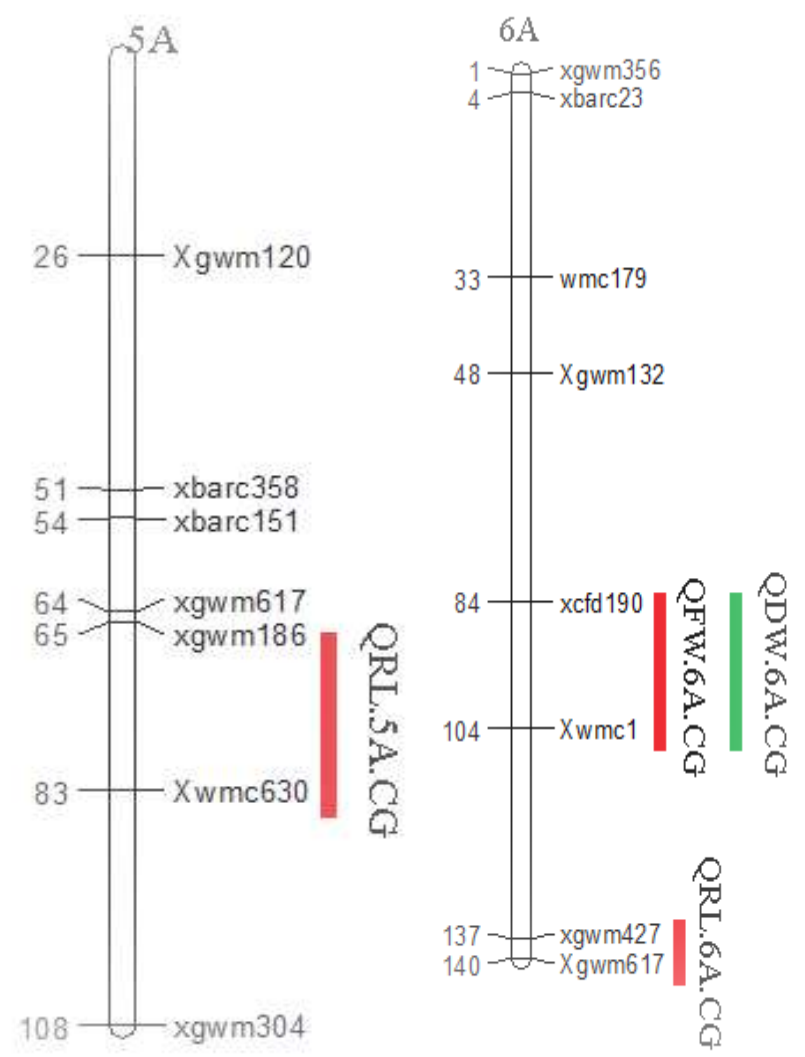

Figure 9. QTL identified on $5 \mathrm{~A}$ and $6 \mathrm{~A}$ chromosome of recombinant inbred lines in non-stressed and stressed conditions. 

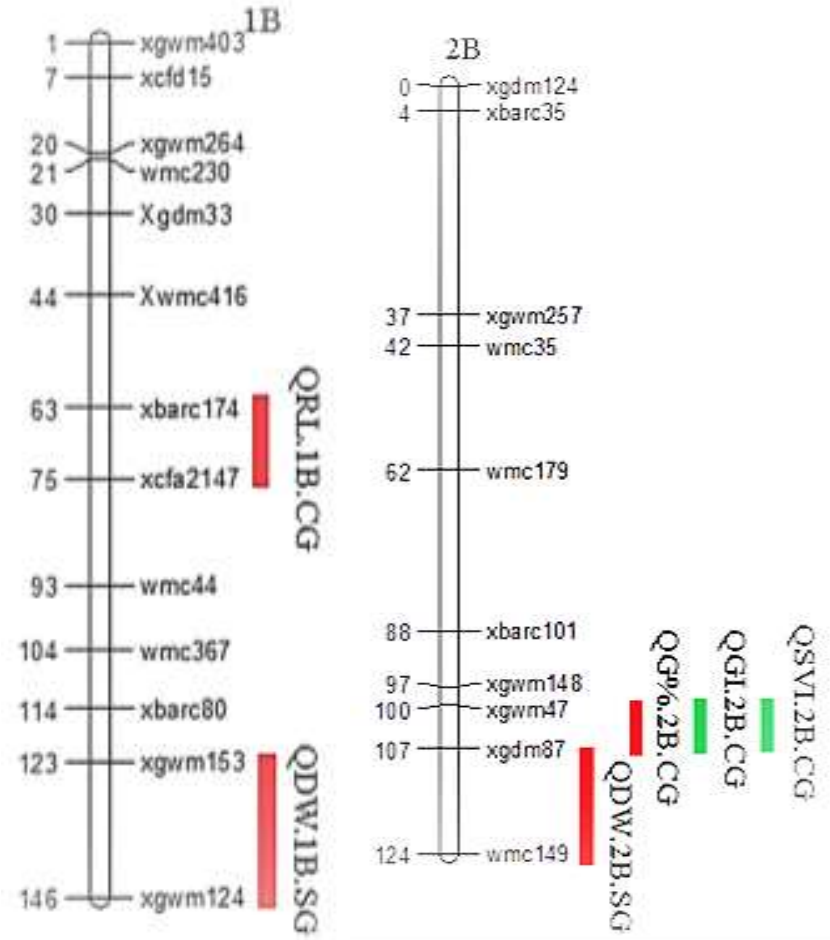

Figure 10. QTL identified on 1B and 2B chromosome of recombinant inbred lines in non-stressed and stressed conditions.

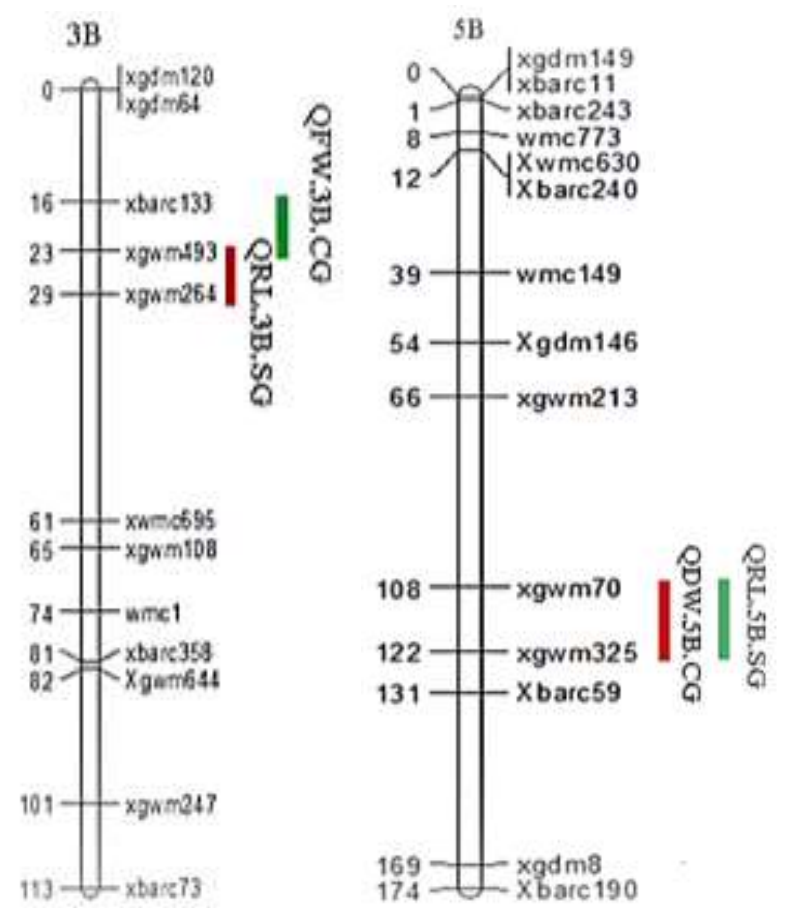

Figure 11. QTL identified on $3 B$ and $5 B$ chromosome of recombinant inbred lines in non-stressed and stressed conditions.

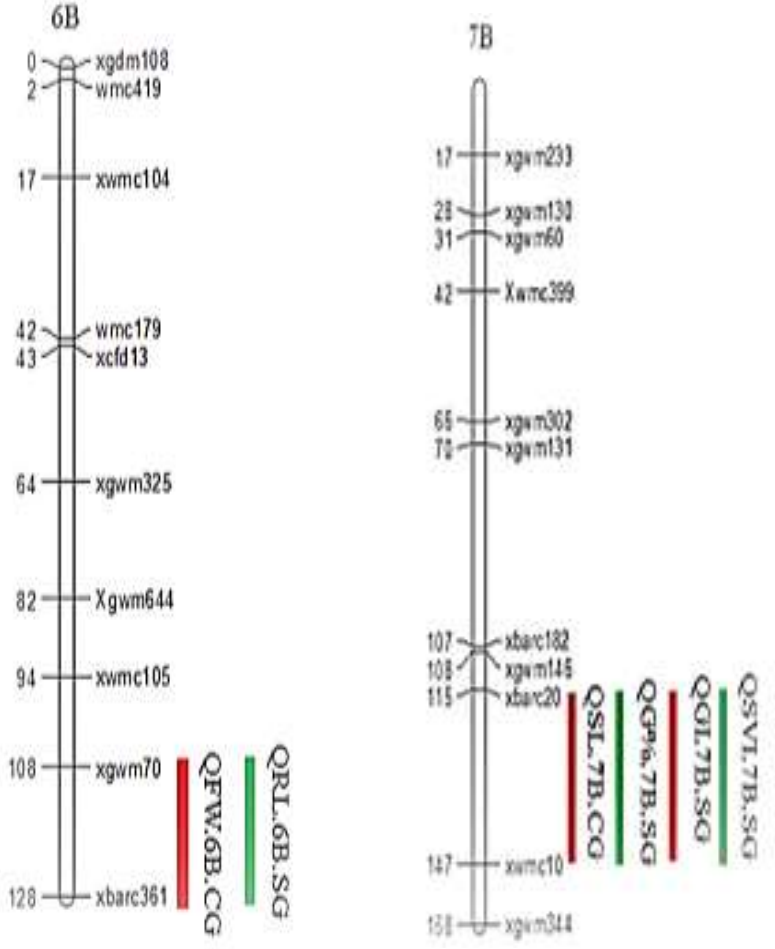

Figure 12. QTL identified on 6B and 7B chromosome of recombinant inbred lines in non-stressed and stressed conditions.
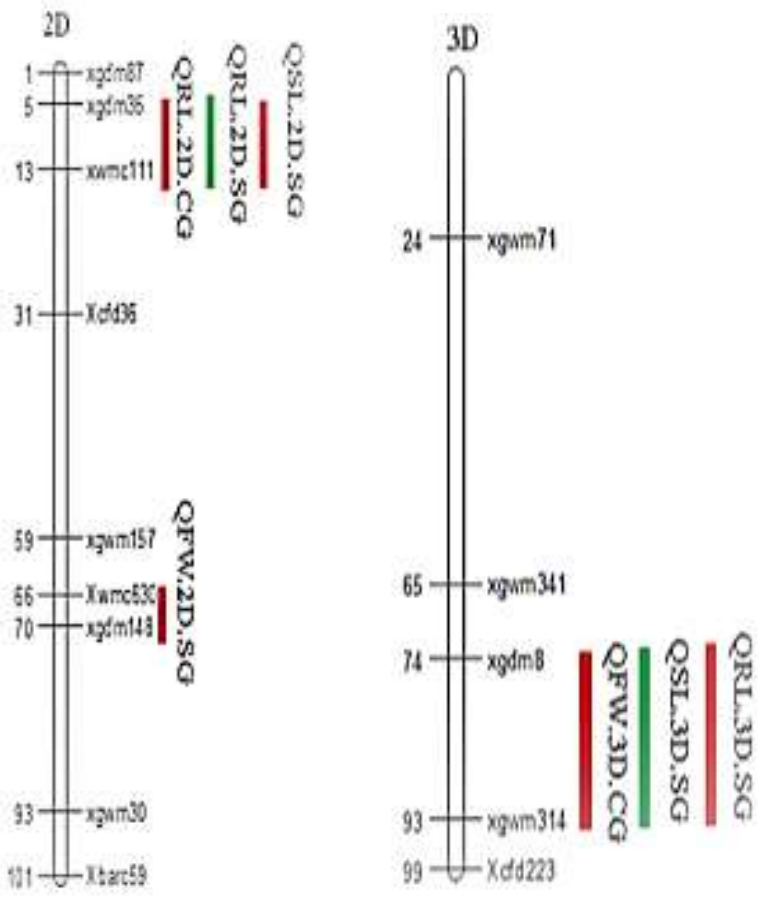

Figure 13. QTL identified on 2D and 3D chromosome of recombinant inbred lines in non-stressed and stressed conditions. 


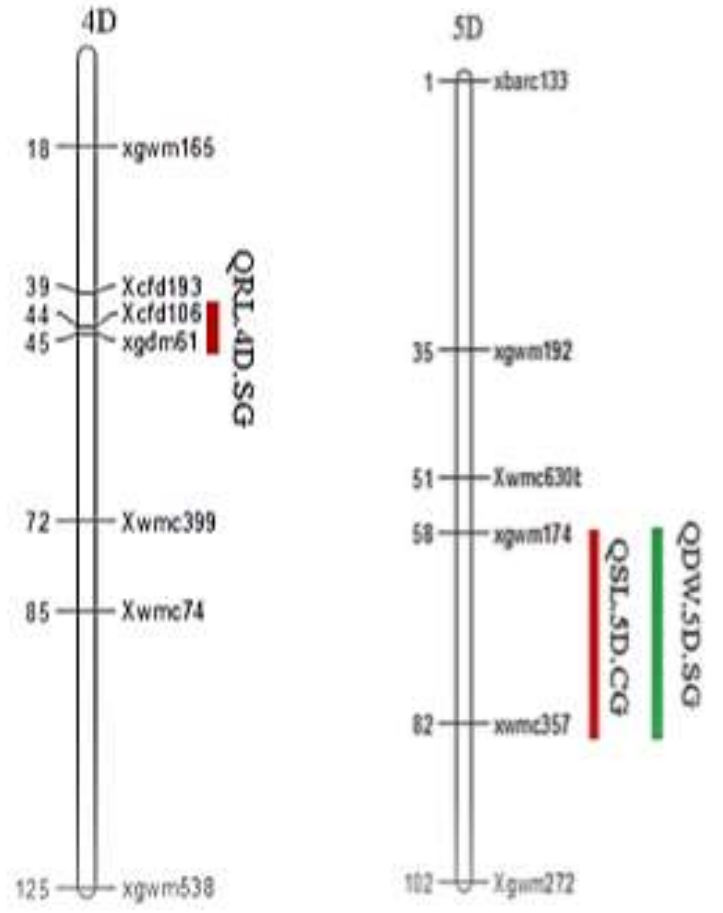

Figure 14. QTL identified on 4D and 5D chromosome of recombinant inbred lines in non-stressed and stressed conditions.

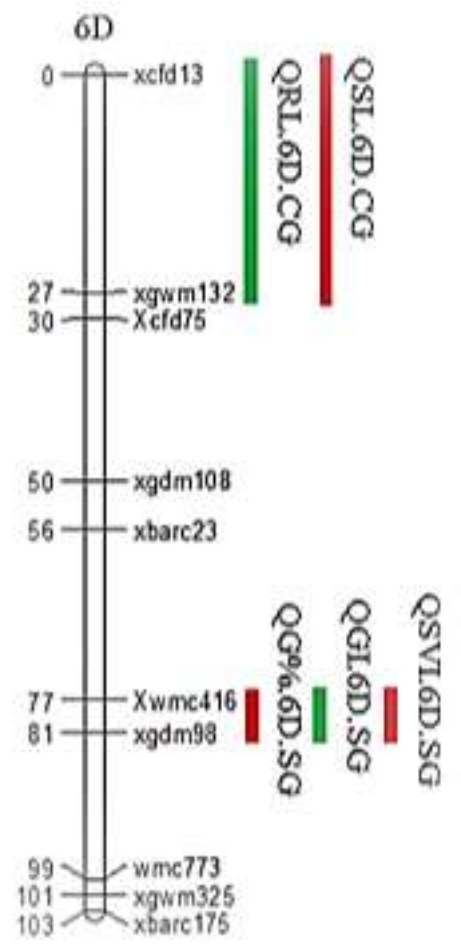

Figure 15. QTL identified on 6D chromosome of recombinant inbred lines in non-stressed and stressed conditions.
QTL for germination attributes: QTL (QG\%.2B.CG) for germination percentage was localized on $2 \mathrm{~B}$ chromosome of recombinant inbred lines under control condition and contributes $13 \%$ of phenotypic variation. In salt stress treatment $(150 \mathrm{mM})$, QTLs for germination percentage $(\mathrm{G} \%)$ were mapped on 2A, 4A, 7B and 6D chromosomes (Table 2). One major QTL (QG\%.4A.SG) for germination percentage was mapped on 4A chromosomes having 14 percent phenotypic variation.

Table 2. Quantitative trait loci (QTL) was associated with traits of RILs mapping population.

\begin{tabular}{|c|c|c|c|c|}
\hline $\begin{array}{l}\text { Quantitative } \\
\text { trait loci (QTL) }\end{array}$ & Markers Interval & LOD & $\begin{array}{c}\text { Phenotypic } \\
\text { variation }\end{array}$ & $\begin{array}{l}\text { Add. } \\
\text { Eff. }\end{array}$ \\
\hline QG\%.2B.CG & xgwm47-Xgdm87 & 2.6 & 13.00 & 1.500 \\
\hline QG\%.2A.SG & wmc177-xbarc199 & 2.8 & 14.01 & -3.370 \\
\hline QG\%.4A.SG & xgwm160-xwmc219 & 2.9 & 14.01 & 1.900 \\
\hline QG\%.6D.SG & xwmc416-xgdm98 & 2.6 & 13.00 & 3.100 \\
\hline QG\%.7B.SG & Xbarc20-Xwmc10 & 2.6 & 13.00 & 3.000 \\
\hline QGI.2B.CG & xgwm47-Xgdm87 & 2.5 & 13.00 & 0.990 \\
\hline QGI.2A.SG & wmc177-xbarc199 & 2.7 & 13.00 & -0.340 \\
\hline QGI.4A.SG & xgwm160-xwmc219 & 3.1 & 15.00 & -0.100 \\
\hline QGI.6D.SG & xwmc416-xgdm98 & 2.6 & 13.00 & 0.310 \\
\hline QGI.7B.SG & Xbarc20-Xwmc10 & 2.5 & 12.00 & 0.300 \\
\hline QSVI.2B.CG & xgwm47-Xgdm87 & 2.6 & 13.00 & 0.010 \\
\hline QSVI.2A.SG & wmc 177 -xbarc199 & 2.7 & 13.00 & 0.340 \\
\hline QSVI.4A.SG & xgwm160-xwmc219 & 3.1 & 15.00 & -0.100 \\
\hline QSVI.6D.SG & xwmc416-xgdm98 & 2.6 & 13.00 & 0.030 \\
\hline QSVI.7B.SG & Xbarc20-Xwmc10 & 2.6 & 12.00 & 0.020 \\
\hline QR1.1B.CG & xbarc174-xcfa2147 & 2.8 & 14.01 & 0.100 \\
\hline QR1.2A.CG & Wmc177-xbarc199 & 2.6 & 13.00 & 0.100 \\
\hline QR1.2D.CG & xgdm35-xwmc111 & 2.6 & 12.00 & -0.900 \\
\hline QR1.6A.CG & xgwm 427 & 3.1 & 15.00 & -0.200 \\
\hline QR1.5A.CG & Xgwm186-Xwmc630 & 2.6 & 13.00 & -0.730 \\
\hline QR1.6D.CG & Xcfd13-xgwm132 & 3.6 & 17.00 & -0.420 \\
\hline QR1.2D.SG & xgdm35-xwmc111 & 3.9 & 19.00 & -2.810 \\
\hline QR1.3B.SG & Xgwm493-Xgwm264 & 3.4 & 16.00 & 0.190 \\
\hline QR1.5B.SG & xgwm70 & 2.7 & 13.00 & 0.260 \\
\hline QR1.6B.SG & Xgwm70-Xbarc361 & 3.2 & 16.00 & 0.200 \\
\hline QR1.3D.SG & xgdm8-xgwm314 & 3.2 & 16.00 & 0.050 \\
\hline QR1.4D.SG & xcfd106-xgdm61 & 2.6 & 13.00 & 0.010 \\
\hline QS1.6D.CG & Xcfd13-xgwm132 & 3.3 & 16.00 & -0.370 \\
\hline QS1.7B.CG & Xbarc20-Xwmc10 & 2.8 & 14.01 & 0.620 \\
\hline QS1.5D.CG & Xgwm174-Xwmc357 & 3.0 & 15.00 & -0.100 \\
\hline QS1.2D.SG & xgdm35-xwmc111 & 2.6 & 13.00 & 0.010 \\
\hline QS1.3D.SG & xgdm8-xgwm314 & 2.9 & 14.01 & -0.100 \\
\hline QFw.3B.CG & xbarc133-xgwm493 & 3.0 & 15.00 & 0.028 \\
\hline QFw.3D.CG & xgdm8-xgwm314 & 2.6 & 13.00 & 0.020 \\
\hline QFw.6A.CG & Xcfd190-Xwmc1 & 2.6 & 13.00 & -0.080 \\
\hline QFw.6B.CG & Xgwm70-Xbarc361 & 3.3 & 16.00 & 0.042 \\
\hline QFw.1D.SG & xbarc66-xbarc346 & 2.8 & 14.01 & 0.010 \\
\hline QFw.2A.SG & Xgwm356-xgwm30 & 2.6 & 13.00 & 0.014 \\
\hline QFw.2D.SG & xwmc630-xgdm148 & 3.1 & 15.00 & 0.010 \\
\hline QDw.5B.CG & Xgwm70-xgwm325 & 2.6 & 13.00 & 0.020 \\
\hline QDw.6A.CG & Xcfd190-xwmc1 & 4.1 & 20.00 & -0.040 \\
\hline QDw.1B.SG & Xgwm153-Xgwm124 & 2.6 & 13.00 & -0.200 \\
\hline QDw.2B.SG & Xgdm87-Xwmc149 & 2.6 & 13.00 & 0.010 \\
\hline QDw.5D.SG & Xgwm174-Xwmc357 & 2.6 & 13.00 & -0.050 \\
\hline
\end{tabular}


One major and three minor QTLs were mapped on 2A, 4A, $7 \mathrm{~B}$ and $6 \mathrm{D}$ chromosome of RILs for germination index (GI) in $150 \mathrm{mM}$ salt level. Germination index QTLs with LOD value 2.5 to 3.1 contributes about 13 to $15 \%$ in phenotypic variation (Table 2). Positive alleles for these QTLs are from Pasban-90. In control condition one QTL for germination index (GI) was identified on 2B. QTLs for seedling vigor index were identified on $2 \mathrm{~A}, 4 \mathrm{~A}$ and $7 \mathrm{~B}$ chromosome of recombinant inbred lines under salt stress. Seedling vigor index QTL (QSVI.4A.SG) was mapped on 4A with LOD 3.1 and contributes phenotypic variation fifteen percent (Table 2). QTL for seedling growth: QTLs for root length were mapped on chromosome $3 \mathrm{~B}, 5 \mathrm{~B}, 6 \mathrm{~B}, 2 \mathrm{D}, 3 \mathrm{D}$ and $4 \mathrm{D}$ of RILs in stressed treatment. Both parents donate positive alleles for these QTLs and contribute thirteen to nineteen percent phenotypic variation. One major QTL for root length (QRL.2D.SG) was localized on 2D chromosome and 3.9 LOD value. Pasban90 contributes positive allele for this QTL and explained $19 \%$ of phenotypic variation. In control condition, for root length six QTLs were mapped on 2A, 5A, 6A, 1B, 2D and 6D chromosome of RILs. QTL (QRL.6D.CG) was identified as major QTL for root length on 6D chromosome in control condition. This QTL was identified with 3.5 LOD value and $17 \%$ phenotypic variation (Table 2 ).

QTLs were mapped on chromosome 2D, 3D, and 4D of RILs for shoot length in stressed environment. For shoot length one major QTL was identified on 3D chromosome and having fourteen percentage of phenotypic variation. Three QTLs for shoot length are identified in control condition, out of three one major and two minor QTLs were mapped on 7B, 5D and 6D chromosomes of RILs. QTLs for shoot length showed 13 to $16 \%$ phenotypic variation. QTL (QSL.6D.CG) were mapped on 6D chromosome recognized as a major QTL for shoot length with LOD of 3.2 and are found in interval of Xcfd13-xgwm132.

QTLs for seedling fresh weight in stressed treatment were mapped on 2A, 1D and 2D chromosome of RILs. One major QTL for fresh weight having 16 percentage of phenotypic variation was localized on $2 \mathrm{D}$ chromosome and this QTL flanked between SSR markers xwmc630-xgdm148. QTLs for fresh weight were mapped on $6 \mathrm{~A}, 3 \mathrm{~B}, 6 \mathrm{~B}$ and $3 \mathrm{D}$ chromosome of RILs in non-stressed environment. Fresh weight QTLs contributes 13 to $16 \%$ phenotypic variation. QTL for fresh weight was mapped on 6B was considered major QTL with 3.2 LOD. This QTL was found in interval of SSR markers Xgwm70-Xbarc361 and Pasban 90 and contributes positive allele for this QTL.

QTL for dry weight were mapped on 1B, 2B and 5D chromosomes of recombinant inbred lines in stressed environment (Table 2). One major QTL for fresh weight in salt stress was mapped on $2 \mathrm{~B}$ and flanked between SSR markers Xgdm87-xwmc149. Dry weight QTL was mapped on $6 \mathrm{~A}$ and $5 \mathrm{~B}$ chromosome of RILs in non-stressed environment (Table 2). Dry weight QTL explained 13 to 20 percentages of phenotypic variation and dry weight QTL was identified on 6A chromosome of RILs considered a major QTL for dry weight. This QTL was identified with 4.1 LOD and $20 \%$ of phenotypic variation.

Germination attributes QTLs were segregated with QTLs of early seedling growth (Fig. 8-15). Germination percentage QTLs was co-segregated with germination index and seedling vigor index QTLs on 4A, 2B and 6D chromosome of RILs. Germination attributes QTLs were co-segregated with root length and shoot length QTL on 2A and 7B chromosome of RILs. QTL on 2A chromosome flanked in interval of SSR markers xwmc177-xbarc199. Fresh weight and dry weight QTL in control condition shared location on 6A chromosome in interval xcfd190-xwmc1. QTLs of root length co segregated with dry weight, fresh weight and shoot length QTLs on 5B, 6B and 2D chromosome of RILs. Root length QTLs shared location on 2D chromosome under stressed and non-stressed condition. Shoot length QTLs co segregated with dry weight and root length on 5D and 6D of chromosome of recombinant inbred lines.

\section{DISCUSSION}

Present study suggests a significant correlation between germination attributes and seedling vigor index. Our results are similar to Arraouadi et al. (2012) findings, who observed a significant correlation among germination percentage and seedling parameters (dry weight and root length). The significant transgressive segregation were recorded for all trait of RILs generated from cross between Pasban90 and Frontana. Ghaedrahmati et al. (2014) recorded similar trend in which wheat mapping population (RILs) display transgressive segregation for all observed traits in stressed environment. Parents lies far from each other in distribution under salt stress (Czyczyło-Mysza et al., 2014; Azadi et al., 2015).

Current study finding prove that wheat seed germination $\&$ seedling growth phase is polygenic nature and many genes involved. Germination percentage QTL in stressed condition was localized on 4A chromosome recognized as major QTL and QTL flanked in interval xgwm160-xwmc219. Similar findings by Czyczyło-Mysza et al. (2014), recorded germination percentage QTLs in non-stressed and stressed condition on chromosome $5 \mathrm{~A}, 1 \mathrm{~B}, 3 \mathrm{~B}, 4 \mathrm{~B}$ and $6 \mathrm{~B}$ of mapping population (double haploid lines). Likewise, QTLs for germination percentage were mapped on $2 \mathrm{~A}$ and $4 \mathrm{~B}$ chromosome of recombinant inbred lines (Marouf and Mohammadi, 2015) and QTLs for germination percentage was identified in non-stressed and stressed environment (Wang et al., 2011). QTLs for seedling vigor index and germination index were localized on chromosome $2 \mathrm{~A}, 4 \mathrm{~A}$, $2 \mathrm{~B}, 7 \mathrm{~B}$ and $6 \mathrm{D}$ of RILs under control and salt stress. Germination attributes QTLs shared location in interval of SSR markers Xwmc177-Xbarc199. Similarly, Germination 
rate index QTLs were mapped on chromosome 2A and 4B of mapping population (Marouf and Mohammadi, 2015). Wheat seed germination attributes QTLs were mapped on chromosome 1D, 5D and 7D (Landjeva et al., 2014).

QTLs affecting seedling growth was mainly mapped on chromosome 1B, 2A, 2B, 2D, 3B, 3D,4D, 5A, 5B, 5D, 6A, $6 \mathrm{~B}, 6 \mathrm{D}$ and $7 \mathrm{~B}$ of recombinant inbred lines under control and salt stress condition. QTLs for root length were mapped on $1 \mathrm{~B}, 2 \mathrm{~A}, 2 \mathrm{D}, 3 \mathrm{~B}, 3 \mathrm{D}, 4 \mathrm{D}, 5 \mathrm{~A}, 5 \mathrm{~B}, 6 \mathrm{~A}, 6 \mathrm{~B}$, and $6 \mathrm{D}$ chromosome of wheat RILs under non-stressed and stressed condition. Likewise, Czyczyło-Mysza et al. (2014) conducted study to identify QTLs for root length in wheat mapping population in non-stressed and stressed condition. They mapped root length QTLs on chromosome 1A, 2A, 2B, 5B, $7 \mathrm{~B}, 2 \mathrm{D}, 4 \mathrm{D}$ and $6 \mathrm{D}$ of mapping population. In different salt levels, Salem and Magdy (2014) mapped QTLs for root length on 1D, 2D, 3A, 3B, 4B, 5B, 7A and 7B chromosome. Bai et al. (2013) finding suggests that QTLs localized on 6A, 3B, 5B and 4D chromosome for root length. QTLs for shoot length were identified on $1 \mathrm{D}, 2 \mathrm{D}, 3 \mathrm{~B}, 3 \mathrm{D}, 4 \mathrm{~B}, 5 \mathrm{D}, 6 \mathrm{D}$ and $7 \mathrm{~B}$ chromosome of wheat recombinant inbred lines under normal and $150 \mathrm{mM}$ salt stress. QTls for shoot length was reported under non-stressed and stressed condition. Shoot length QTLs were mainly mapped on wheat D genome (Salem and Magdy, 2014). Similarly, another study conducted by Marjaei et al. (2014), there finding depict that shoot length QTLs were mapped on 2D, 3B and 5D chromosome of RILs. Fresh weight QTLs were mapped on 1D, 2A, 2D , 4B, 4D, 5D, 7A and 7D chromosome of RILs in both normal and stressed environment. QTL for fresh weight explained phenotypic variation 15 percentage and found between SSR markers xwmc630-xgdm148. Similar, Salem and Magdy (2014) conducted study to identify QTLs for fresh weight under control and salt stress condition. They mapped QTLs on chromosome 4D, 5A, 5D, 7A and 7D of mapping population. Dry weight QTLs were localized on chromosome 1B, 2B, 5B, $5 \mathrm{D}$ and $6 \mathrm{~A}$ of recombinant inbred lines under non-stressed and stressed condition. Our findings are similar to Salem and Magdy (2014) study, in which they used different salt levels such as $15 \mathrm{mM}, 250 \mathrm{mM}$ and $350 \mathrm{mM}$. Dry weight QTLs were mapped on 1D, 3B, 4B, 4D, 5A, 7A and 7D chromosome of RILs. Czyczyło-Mysza et al. (2014) findings depict that dry weight QTLs were mapped on chromosome 2B, 3B, 6B, 5B, $5 \mathrm{D}, 6 \mathrm{~B}, 6 \mathrm{D}$ and $7 \mathrm{~A}$ of mapping population

Conclusion: Current study findings show that wheat seed germination and early seedling growth is poly genic trait. QTLs for seed germination and seedling growth are collocated at same in locus interval and this show a main mechanism for salt tolerance.

\section{REFERENCES}

Abdulbaki, A. and J.D. Anderson. 1973. Vigor determination in Soybean seed by multiple criteria. Crop Sci. 13:630633.

Ahmad, M., A. Shahzad, M. Iqbal, M. Asif and H.H. Arvind. 2013. Morphological and molecular genetic variation in wheat for salinity tolerance at germination and early seedling stage. Aust. J. Crop Sci. 7:66-74.

Amin, Y.A. and A.D. Ayman. 2013. QTL mapping of wheat (Triticum aestivum L.) in response to salt stress. Int. J. Bio. Tech. 3:47-60.

Arraouadi, S., M. Badri, C. Abdelly, T. Huguet and M.E. Aouani. 2012. QTL mapping of physiological traits associated with salt tolerance in Medicago truncatula recombinant inbred lines. Genet. 99:118-125. 10.1016/j.ygeno.2011.11.005.

Azadi, A., M. Mohsen, M.H. Eslam, A.M. Seyed, M. Foad, I.T. Mohammad, M.P. Seyed, E. Mohsen, F. Farzad, K. Mehrbano, A. Sadegh, N. Babak and M.N. Ghasem. 2015. QTL mapping of yield and yield components under normal and salt-stress conditions in bread wheat (Triticum aestivum L.). Plant Mol. Biol. Rep. 33:102120. DOI 10.1007/s11105-014-0726-0.

Bai, C., Y. Liang and M.J. Hawkesford. 2013. Identification of QTLs associated with seedling root traits and their correlation with plant height in wheat. J. Exp. Bot. 64:1745-1753.

Batool, N., N. Ilyas, A. Shahzad, M. Arshad and F.U. Hassan. 2016. Evaluation of wheat genotypes on the basis of physiological indices under salt stress. Pure Appl. Biol. 5:1183-1192.

Czyczylo-Mysza, I., M. Izabela, S. Edyta, C. Katarzyna, J. Katarzyna and K. Malgorzata. 2014. QTL mapping for germination of seeds obtained from previous wheat generation under drought. Cent. Eur. J. Biol. 9:374-382. DOI: 10.2478/s11535-013-0273-y.

EL-Hendawy, S.E., Y. Ruan, Y. Hu and U. Schmidhalter. 2009. A comparison of screening criteria for salt tolerance in wheat under field and controlled environmental conditions. J. Agro. Crop Sci. 195:356367.

Genc, Y., K. Oldach, A. Verbyla, G. Lott, M. Hassan, M. Tester, H. Wallwork and G.K. McDonald. 2010. Sodium exclusion QTL associated with improved seedling growth in bread wheat under salinity stress. Theor. Appl. Genet. 121:877-894.

Ghaedrahmati, M., M. Mardi, M.R. Naghavi, E. Majidi Haravan, B. Nakhoda, A. Azadi and M. Kazemi. 2014. Mapping QTLs associated with salt tolerance related traits in seedling stage of wheat (Triticum aestivum L.). J. Agric. Sci. Technol. 16:1413-1428.

Gupta, N.K., S. Gupta and A. Kumar. 2001. Effect of water stress on physiological attributes and their relationship with growth and yield of wheat cultivars at different stages. J. Agron. Crop Sci. 186:55-62. 
Guyomarc'h, H., P. Sourdille, G. Charmet, K.J. Edwards and M. Bernard. 2002. Characterization of polymorphic microsatellite markers from Aegilops taushii and transferability to the D-genome of bread wheat. Theor. Appl. Genet. 104:1164-1172.

ISTA. 2005. International rules for seed testing. International Seed Testing Association. Zurich, Switzerland.

Landjeva, S., U. Lohwasser and A. Borner. 2010. Genetic mapping within the wheat D genome reveals QTL for germination, seed vigour and longevity, and early seedling growth. Euphytica 171:129-143.

Ma, L., E. Fengzhou and N. Huo. 2007. Genetic analysis of salt tolerance in a recombination inbred population of wheat. Euphytica 153:109-117.

Mahdinejad, N., M. Omidi, M.R. Jalalkamali, M.R. Naghavi and B.A. Fakheri. 2014. QTL analysis of some phenological and morphological traits in Babax and Seri M82 recombinant inbred line population of wheat during salinity stress. Modern Genet. 9:207-218.

Marjaei, H.S., G.A. Ranjbar and A.M. Naji. 2014. Evaluation of QTL associated with salt tolerance on seedling stage in wheat. Inter. J. Biosci. 4:163-168.

Marouf, K. and S.A. Mohammadi. 2015. Mapping qtls associated with wheat seed germination under normal and drought stress conditions. J. crop Biotech. 4:1-14.

Mirza, S.R., N. Ilyas and N. Batool. 2015. Seed priming enhanced seed germination traits of wheat under water, salt and heat stress. Pure Appl. Biol. 4:650-658.

Munns, R. and M. Tester. 2008. Mechanisms of salinity tolerance. Ann. Rev. Plant Biol. 59:651-681.

Munns, R. and R.A. James. 2003. Screening methods for salt tolerance: A case study with tetraploid wheat. Pl. Soil 253:239-250.
Nichols, P.G.H., A. Malik and M. Stockdale. 2009. Salt tolerance and avoidance mechanisms at germination of annual pasture legumes: importance for adaptation to saline environments. P1. Soil 315:241-255.

Roder, M.S., V. Korzun, K. Wendehake, J. Plaschke, M.H. Tixier, P. Leroy and M.W. Ganal. 1998. A microsatellite map of wheat. Genetics 149:2007-2023.

Salem, K.F.M. and Z.M. Magdy. 2014. Identification of microsatellite alleles for salt tolerance at seedling stage in wheat (Triticum aestivum L.). Life Sci. J. 11:10641073.

Shahzad, A., M. Ahmad, M. Iqbal, I. Ahmed and G.M. Ali. 2012. Evaluation of wheat landrace genotypes for salinity tolerance at vegetative stage by using morphological and molecular markers. Genet. Mol. Res. 11:679-692.

Sharp, P.J., S. Desai, S. Chao and M.D. Gale. 1988. Isolation and use of a set of 14 RFLP probes for the identification of each homeologous arm in the Triticeae. In: T.E. Miller and R.M.D. Koebner (eds.), Proc. $7^{\text {th }}$ Int. Wheat Gen. Symp. IPSR. Cambridge Lab, Cambridge, UK. pp.639643.

Somers, J.D., P. Isaac and K. Edwards. 2004. A high density microsatellite consensus map for bread wheat (Triticum aestivum L.). Theor. Appl. Genet. 109:1105-1114.

Song, Q.J., J.R. Shi, S. Singh, E.W. Fickus, J.M. Mosta, J. Lewis, B.S. Gill, R. Ward and P.B. Cregan. 2005. Development and mapping of microsatellite (SSR) markers in wheat. Theor. Appl. Genet. 110:550-560.

Wang, Z.F., J.F. Wang, Y.M. Bao, Y.Y. Wu and H.S. Zhang. 2011. Quantitative trait loci controlling rice seed germination under salt stress. Euphytica 178:297-307. 